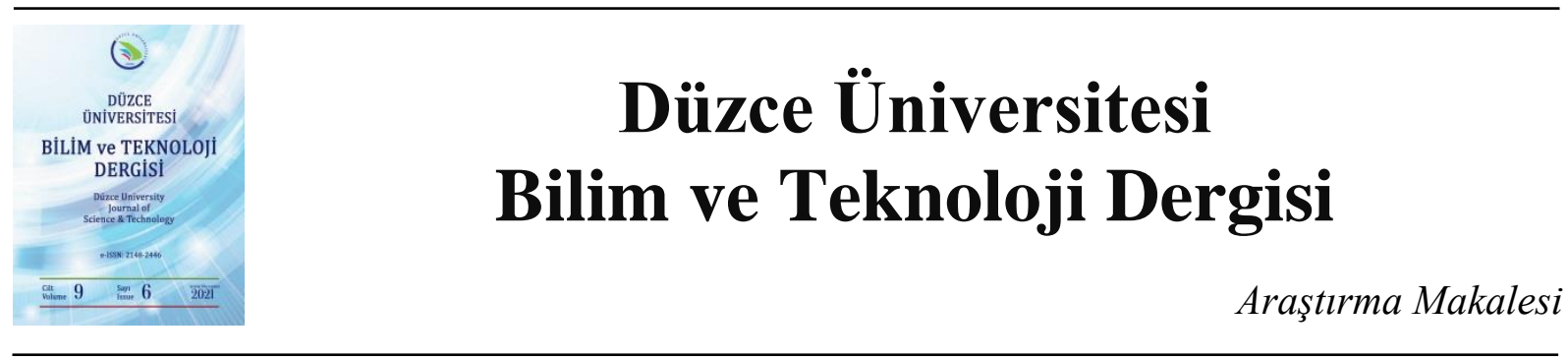

\section{Üstverilerin Derin Öğrenme Algoritmaları Kullanılarak Otomatik Olarak Çıkartılması ve Sınıflanması ${ }^{1}$}

\author{
(iD) Murat İNCE ${ }^{\mathrm{a}, *}$ \\ ${ }^{a}$ Bilgisayar Teknolojileri Bölümü, TBMYO, Isparta Uygulamalı Bilimler Üniversitesi, Isparta, TÜRKIYYE \\ * Sorumlu yazarin e-posta adresi: muratince@isparta.edu.tr \\ DOI:10.29130/dubited.1012046
}

\begin{abstract}
$\ddot{\mathrm{O} Z}$
Günümüzde bilişim teknolojilerinin yaygınlaşması sebebiyle dijital içerik ihtiyacı artmıştır. Bu içeriklerin oluşturulması zaman alıcı ve maliyetli bir süreçtir. İçerik oluşturulurken öğrenme nesnelerinden faydalanılmaktadır. Bu nesnelerin bilgisayarlar tarafından keşfedilebilir ve okunabilir olması yeniden kullanılabilirlik ve paylaşılabilirlik açısından önemlidir. Bu sebeple nesneler tanımlayıcı kimlik bilgilerini içeren üstveriler ile bütünleşik olarak kullanılmaktadırlar. Bu üstveriler ne kadar düzgün oluşturulup sınıflandırılırsa nesnelerin kullanılabilirliği o derece artmış olmaktadır. Bu sebeple nesnelerden otomatik üstveri çıkartan birçok yöntem geliştirilmiştir. Bu çalışmada da Konvolüsyonel Sinir Ağları (KSA), Tekrarlayan Sinir Ağları (TSA) gibi derin öğrenme ve Doğal Dil İşleme (DDİ) yöntemleri kullanılarak öğrenme nesnelerindeki içeriklerden otomatik olarak üstveri çıkartılması ve sınıflaması yapılmışıı. Sistemin başarısı ve doğruluğu örnek öğrenme nesneleri ile test edilmiş̧ir. Sonuçlar sistemin başarılı bir şekilde kullanılabileceğini göstermiştir.
\end{abstract}

Anahtar Kelimeler: Üstveri çıkartma, Konvolüsyonel sinir ă̆ları, Tekrarlayan sinir ağları

\section{Automatic Metadata Extraction and Classification by using Deep Learning Algorithms}

\begin{abstract}
The need for digital content has increased due to the widespread use of information technologies today. Creating these contents is a time consuming and costly process. Learning objects are used while creating the content. It is important that these objects can be discovered and readable by computers in terms of reusability and shareability. For this reason, objects are used in integration with metadata containing identifying information. The more properly these metadata are created and classified, the greater the usability of the objects. For this reason, many methods have been developed that automatically extract metadata from objects. In this study, metadata extraction and classification from the contents of learning objects were made automatically by using deep learning methods such as Convolutional Neural Networks (CNN), Recurrent Neural Networks (RNN) and Natural Language Processing (NLP). The success and accuracy of the system has been tested with sample learning objects. The results showed that the system can be used successfully.
\end{abstract}

Keywords: Metadata extraction, Convolutional neural networks, Recurrent neural networks

1*ICAIAME 2021 konferansinda sunulmuştur.

Geliş: 19/10/2021, Düzeltme:09/12/2021, Kabul: 12/12/2021 


\section{GIRIS}

Uzaktan eğitim bireylerin kendi kendilerine geleneksel eğitime göre daha esnek olarak öğrenmelerini desteklemektedir [1]. Uzaktan eğitim geleneksel eğitime alternatif olmamakla birlikte yüz yüze eğitim-öğretim olanaklarından kısıtlı kişilere firsat tanımaktadır [2]. $\mathrm{Bu}$ tür eğitimler genellikle internet üzerinden yapılmakta ve web tabanlı eğitim olarak ta tanımlanmaktadır [3]. Günümüzde bilgisayar, tablet ve akıllı telefonların çok sık kullanılması gerek eğitim gerekse eğlence amaçlı birçok içerik ihtiyacına sebep olmuştur [4]. Bu içeriklerin oluşturulması için öğrenme nesnelerinden faydalanılmaktadır [5]. Öğrenme nesnesi; içerik nesnesi, eğitsel nesne, bilgi nesnesi, öğrenme kaynağ1, medya nesnesi ve yeniden kullanılabilir öğrenme kaynağı olarak tanımlanmaktadır [6,7]. Diğer bir tanımla öğrenme nesnesi; eğitsel olarak anlamı olan, sayısal veya sayısal olmayan film, video, ses, animasyon, simülasyon, resim, grafik, metin, harita, kitap gibi kaynaklar olarak tanımlanmaktadır [8]. $\mathrm{Bu}$ nesneler IEEE LOM, IMS, DCMI gibi birçok standart tarafından da desteklenmektedir [9]. Öğrenme nesnelerinde olması gereken özellikler erişilebilirlik, birlikte çalışabilirlik, uyumluluk, yeniden kullanılabilirlik ve değiştirilebilirlik olarak tanımlanmıştır [10,11]. Millar [12] öğrenme nesnesini içeriğin kendisi ve onun tanımlayıcı bilgisini içeren üstveri (metadata) olarak tanımlamıştır. $\mathrm{Bu}$ üstveriler kullanılarak öğrenme nesneleri çok farklı platformlar tarafından desteklenmektedir [13]. Ayrıca üstveri sayesinde bilgisayarlar ve diğer cihazlar tarafından da okunabilirlik sağlanmaktadır.

Öğrenme nesnelerinin içerikleri ve bunların tümleşik üstverileri öğrenme nesne ambarı denilen veritabanlarında saklanmaktadır [14]. İçeriklerin üstverileriyle depolanmaları erişilebilirliği, birlikte çalışabilirliği ve yeniden kullanılabilirliği sağlamaktadır $[15,16]$. Ayrıca bir nesne hem matematik hem fizik hem de diğer başka bir alan için de kullanılabilmektedir. Böylece kaynakların veri tekrarı yapılmadan farklı amaçlar için kullanılması sağlanmakta ve verimlilik artmaktadır [17]. Öğrenme nesne ambarları gerek üzerinde bulunan araçlar gerekse diğer servisler tarafindan üstveri üzerinden sorgulanarak, uygun nesnelerin bulunup birleştirilmesi ve yeni içeriklerin oluşturulması sağlanmaktadır [18]. Bu içerik oluşturma işlemi kullanıcılar tarafından yapılabildiği gibi bilgisayarlar tarafından kullanıcının belirlediği kurallara göre de otomatik olarak yapılabilmektedir. Örneğin; bilgisayar bir metnin görselini dokümanları taratarak oluşturulabilmektedir. Önerilen bu çalışmada sisteme yüklenen web ya da diğer dokümanlardaki resim türündeki içeriklerin derin öğrenme ve doğal dil işleme gibi yapay zeka yöntemleri ile anlamlandırılması ve yorumlanması yapılmaktadır. Elde edilen anlama göre öğrenme nesnesinin üstverileri doldurulmakta ve hangi konu alanı ile olduğu belirlenerek sinıflandırılmaktadır.

Makalenin geri kalanı şu şekilde düzenlenmiştir. Bölüm 2'de kullanılan yöntem ve metotlar açıklanmıştır. Bölüm 3'de önerilen sistem anlatılmıştır. Bölüm 4'de hesaplamalar tartışılmış ve son bölümde sonuçlar verilmiştir.

\section{MATERYAL VE METOT}

\section{A. OTOMATİK ÜSTVERİ OLUSTURMA}

Dijital kaynakların verimli bir şekilde kullanılması için sistemler ve bilgisayarlar tarafindan keşfedilebilir, okunabilir, paylaşılabilir ve değişim yapılabilir olması gerekmektedir [19]. Bu işlemler üstveriler tarafından kolaylıkla sağlanabilmektedir. Kaynak sayısının günümüzde çok fazla olması ve bunların üstverilerinin el ile doldurulmasının oldukça zaman alıcı bir işlem olması sebebiyle otomatik üstveri çıkarma sistemleri geliştirilmiştir [20]. Bu amaçla kural tabanlı ve makine öğrenmesi gibi farklı sistemler kullanılmıştır. Roy vd. [21] öğrenme içeriklerinden pedagojik üstverileri çıkarmışlardır. Cortez vd. [22] bilgi tabanlı veritabanlarından kendi etiketledikleri verileri kıyaslama yaparak üstveri oluşturmuşlardır. Cardineleas vd. [23] ise ARIADNE üzerinde çalışan ve makine öğrenmesi yöntemleri kullanarak IEEE LOM üstverisi dolduran bir sistem geliştirmiş̧lerdir. Han vd. [24] ise destek vektör makinesi ile Dublin Core (DC) üstverilerini doldurmuşlardır. Diğer bir çalışmada 
istatistiki yöntemler kullanılarak metinlerin üstverileri çıkarılmıştır [25]. Bazı çalışmalarda ise ontoloji tabanlı semantik üstveriler oluşturulmuştur [26]. Üstveri çıkarmada kullanılan diğer bir yöntem ise doğal dil işlemedir [27-29]. Bu çalışmalar incelendiğinde webdeki içeriklerden ve nesne ambarlarındaki öğrenme nesnelerinden faydalandıkları görülmektedir. Önerilen çalışmada derin öğrenme yöntemleri ile görüntü işleme, metin oluşturma, doğal dil işleme ve sınıflama gibi birçok yöntem bir arada kullanılarak IEEE LOM üstveri bilgileri doldurulup sınıflanmaktadır.

\section{B. DERİN ÖĞRENME ALGORITTMALARI}

Derin öğrenme algoritmaları günümüzde görüntü işleme, sınıflandırma, doğal dil işleme gibi birçok alanda sıklıkla kullanılmaktadır [30]. Derin öğrenme ağları da diyebileceğimiz bu yöntemler klasik yapay sinir ağlarından katman sayılarının uygulanışı gibi birkaç yönden farklılık göstermektedir [31]. Konvolüsyonel (Evrişimli) Sinir Ağı (KSA) en bilinen derin öğrenme algoritmalarından biridir [32,33]. KSA ileri beslemeli sinir ağı olmasına karşın evrişim, ortaklama, tam bağlantı gibi birçok başka katman içermektedir [34]. KSA en çok görüntü işlemede resimlerin boyutlarının düşürülmesi, özniteliklerin çıkarılması ve sınıflandırılması amacıyla kullanılmaktadır [35]. Diğer sık kullanılan derin öğrenme algoritması ise Tekrarlayan Sinir Ağı (TSA)'dır [36]. TSA'lar aynı fonksiyonu her bir giriş için uygularken bir önceki çıkışta hesaplanan değeri ilişkilendirmektedir [37,38]. Bir çıkış değeri alınınca bu değer hafızaya kopyalanmakta ve TSA' ya giriş olarak gönderilmektedir. Böylece mevcut işlem adımındaki giriş bir önceki öğrenilen giriş değerine göre hesaplanmaktadır. TSA'lar geri beslemeli ağlardan farklı olarak kendi iç hafızasını kullanarak işlem yapmaktadır [39]. Diğer ağlarda girişler birbirinden bağımsız iken TSA' larda girişler birbirine bağımlıdır. Bu sebeple el yazısı tanıma, ses tanıma, resim etiketleme gibi alanlarda kullanılmaktadır [40,41].

\section{DOĞAL DİL İ̧SEME}

Yapay zekanın popüler uygulamalarından biri olan doğal dil işleme (DDİ), dilin belirli kurallara göre analiz edilip anlanması ve yeniden yapılandırılmasıdır [42]. DDİ işlemleri çoğunlukla metin ön işleme, morfolojik analiz, sözdizimsel analiz ve anlamsal analiz kısımlarını içerir [43]. Morfolojik analiz kısmında kelimelerin eklerinin ayrıştırılarak köklerinin bulunması işlemlerini içerir. Böylece kelimenin isim, sıfat, fiil gibi hangi tipe ait olduğu belirlenir [44]. Söz dizimsel analiz kısmında ise kelimelerin cümle içerisindeki özne, nesne, zarf gibi kullanım amaçları belirlenir [45]. Bu kelimelerin cümlede farklı yerde ve sayıda kullanılması farklı anlamlar içerebilmektedir. Anlamsal analiz ise ayrık kelimelerin uygun nesnelerle ilişkilendirilmesini inceler [46]. Kelimelerin birbirleriyle olan anlamsal bağlantıların ortaya çıkarılması ve kavram haritalarının ortaya çıkarılması sağlanır. Böylece eş anlamlılar, yakın anlamlılar gibi kelimeler belirlenir [47]. Bu işlemlerden sonra metin normalizasyonu yapılır. Bunun için NLTK [48] ve Zemberek [49] gibi doğal dil işleme araçları kullanılarak büyük küçük harf dönüştürme, sayıları metne dönüştürme, noktalama ve işaretlemelerin kaldırılması, boşlukların kaldırılması, kısaltmaların açılması ve gereksiz kelimelerin kaldırılması gibi işıemler uygulanır [50].

\section{PERFORMANS DEĞERLENDİRME METRİKLERİ}

Doğal dil işleme yöntemlerinde oluşturulan ya da çevrilen metinlerin değerlendirilmesi gerekmektedir. Bunun için sık kullanılan yöntemlerden birisi BLEU' dur [51]. Bu yöntemde istenilen referans metin ile test edilecek olan üretilen ya da çevrilen metin arasındaki benzerliğe bakılmaktadır [52]. Metinler arası benzerlik yakınsa değer 1'e yakınlaşmakta, benzerlik uzaksa 0'a yaklaşmaktadır. Bu işlem yapılırken referans metin ile test edilen metin arasındaki n-gramların metinler içerisinde kaçar defa geçtiğinin frekansına göre hesaplanmaktadır. Sınıflandırma sonuçlarının performansının değerlendirilmesinde doğruluk oranı sıklıkla kullanılmaktadır [53]. Söz konusu performans ölçüm parametrelerinden doğruluk oranı değeri, bir testin ilgili sınıfa ait olanları söz konusu sınıfta bulanma oranidir [54]. 


\section{OTOMATIK ÜSTVERİ DOLDURMA VE SINIFLAMA SISTEMI}

$\mathrm{Bu}$ çalışmada sisteme yüklenen öğrenme nesnelerinin içindeki resimlerin ve metinlerin derin öğrenme ve doğal dil işleme yöntemleri ile özelliklerinin çıkarılması, üstverilerinin doldurulması ve ilgili konu alanına göre sınıflandırılmasını sağlayan web tabanlı bir yazılım geliştirilmiştir (Şekil 1). Sisteme yüklenen nesnenin içindeki resim KSA ile kodlanıp, TSA ile de bu kodlanan özelliklerin çözümü ve metinsel olarak karşılığının bulunması sağlanmaktadır. KSA' da resimler giriş katmanında 240 x 240 x 3 olarak kodlanmış ve VGG16 ön eğitim özellikleri kullanılmıştır. Bunun için MS-COCO veri setindeki resimler ve açıklamaları kullanılmıştır. MS-COCO veri seti 300.000 'den fazla resim ve bunların metinsel açıklamalarını içermektedir [55]. Elde edilen metin Google translate servisi ile ilgili dile çevrilmektedir. Daha sonra ise ilgili metin üzerinde temel DDİ yöntemleri uygulanmaktadır. Bunun için Zemberek kütüphanesi kullanılmıştır. Elde edilen metin ile IEEE LOM üstverisine ait ad, açıklama gibi bilgiler otomatik olarak doldurulmaktadır. Ayrıca bu metin sosyal bilimler (Coğrafya, Tarih), fen bilimleri (Fizik, Kimya, Biyoloji, Matematik) alanlarını içeren metinler ile eğitilmiş yeni bir KSA' ya sokularak hangi konu alanına ait olduğu gibi sınıflama bilgileri otomatik olarak doldurulmaktadır. Üstverilerin doğru bir şekilde doldurulması sayesinde öğrenme nesneleri bilgisayarlar ve diğer sistemler tarafından keşfedilebilir, okunabilir ve kullanılabilir olmaktadır.

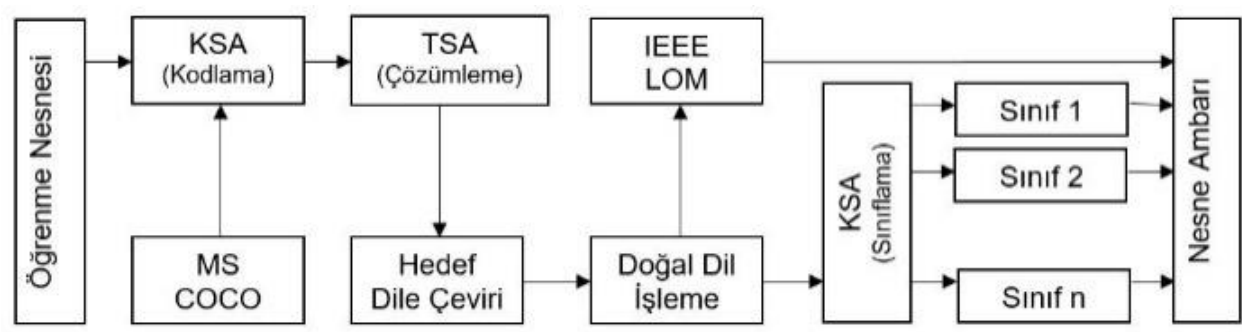

Şekil 1. Üstveri doldurma sistemi şeması

\section{HESAPLAMALAR VE TARTISSMA}

Önerilen otomatik üstveri doldurma ve sınıflama sistemi 130 adet öğrenme nesnesi ile test edilmiştir. Sistem tarafindan resimlere ait olarak oluşturulan metinler Bleu yöntemi ile benzerlik oranları test edilmiştir (Tablo 1). Her konu alanına ait olan öğrenme nesnelerinin Bleu karşılaştırmaları hesaplanmıştır (Şekil 2, Şekil 3, Şekil 4, Şekil 5, Şekil 6, Şekil 7). Sonuçlara bakıldığında en düşük Bleu benzerlik ortalaması Fizik alanı nesnelerinde, en yüksek Bleu benzerlik ortalaması ise Biyoloji alanı nesnelerinde bulunmuştur. Biyoloji alanındaki Bleu puanlarının diğerlerine göre yüksek olmasının sebebi içerdikleri resimlerin MS-COCO veri setindeki resimlere daha çok yakın olmasından kaynaklanmaktadır.

Tablo 1. Nesne sayllarl ve Bleu puanlart

\begin{tabular}{ccc}
\hline Konu & Nesne Sayısı & Bleu Puan Ortalaması \\
\hline Coğrafya & 26 & 0,72996 \\
\hline Tarih & 24 & 0,71563 \\
\hline Fizik & 16 & 0,69772 \\
\hline Kimya & 14 & 0,74464 \\
\hline Biyoloji & 20 & 0,75454 \\
\hline Matematik & 30 & 0,73346 \\
\hline
\end{tabular}




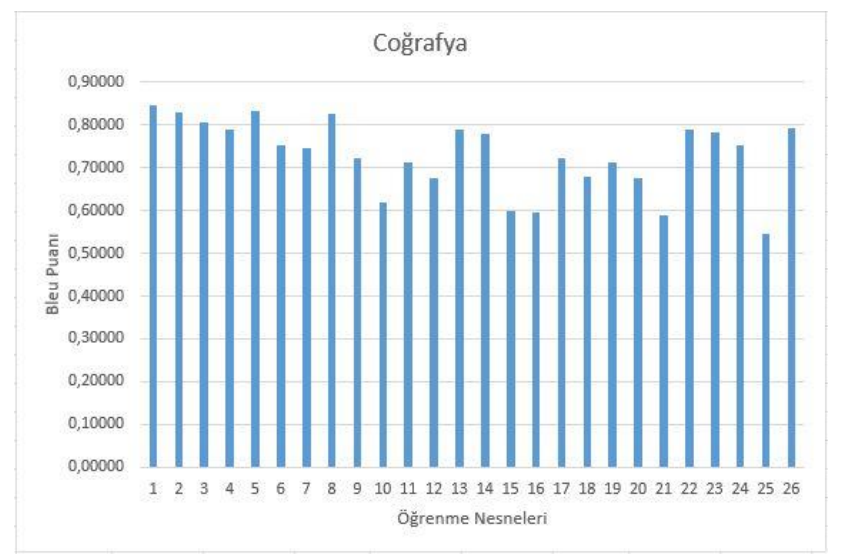

Şekil 2. Coğrafya Bleu puanlart

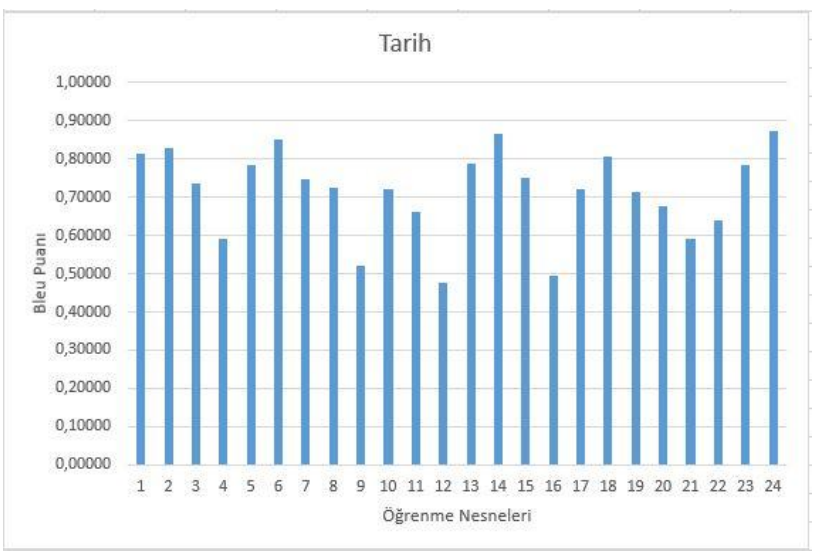

Şekil 3. Tarih Bleu puanları

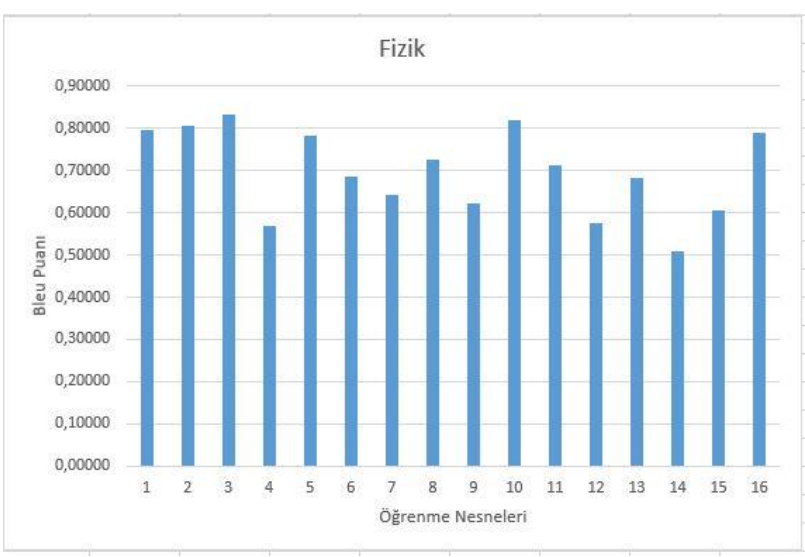

Şekil 4. Fizik Bleu puanları 


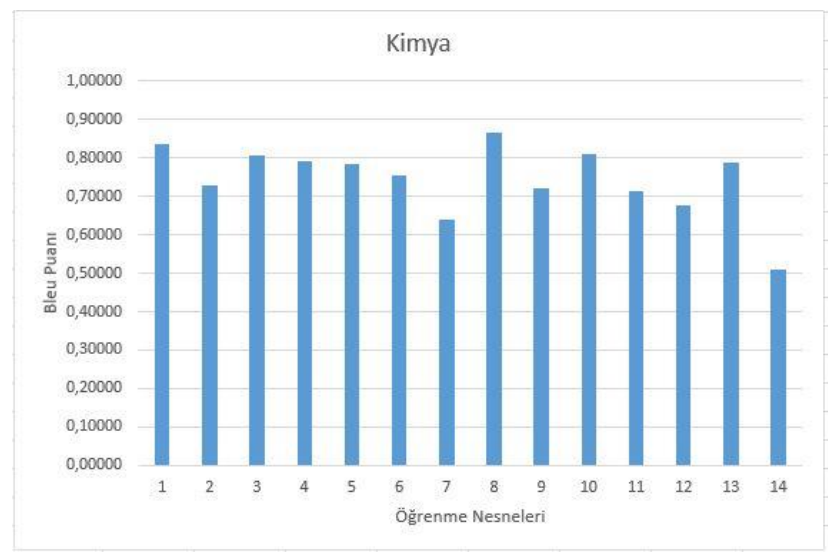

Şekil 5. Kimya Bleu puanları

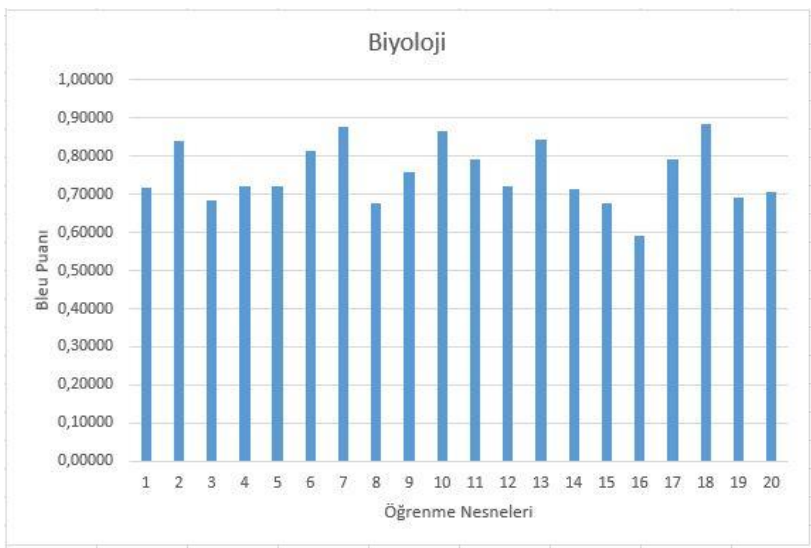

Şekil 6. Biyoloji Bleu puanları

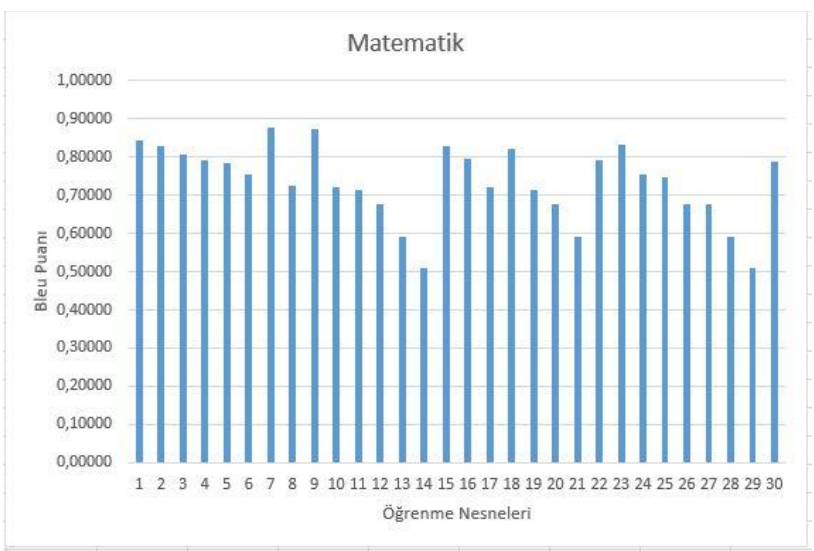

Şekil 7. Matematik Bleu puanlarl

Sistem tarafından oluşturulan metinlerin DDİ yöntemleri sonrasında ilgili konu alanına ait olduğunun belirlenmesi için sınıflandırma işlemi yapılmıştır. Bu sonuçlara ait karışıklık matrisi (Şekil. 8) oluşturulmuştur. Ayrıca doğruluk, kesinlik ve duyarlılık değerleri hesaplanmıştır (Tablo 2). Bu sonuçlara göre en düşük sınıflama doğruluk oranı yine Fizik alanında bulunmuşken, en yüksek sınıflama doğruluk oranı ise yine Biyoloji alanında bulunmuştur. Bleu ve sınıflama oranlarının birbiriyle paralel olması, resimden metin oluşturulma başarısının sınıflama sonuçlarını doğrudan etkilediğini göstermektedir. 


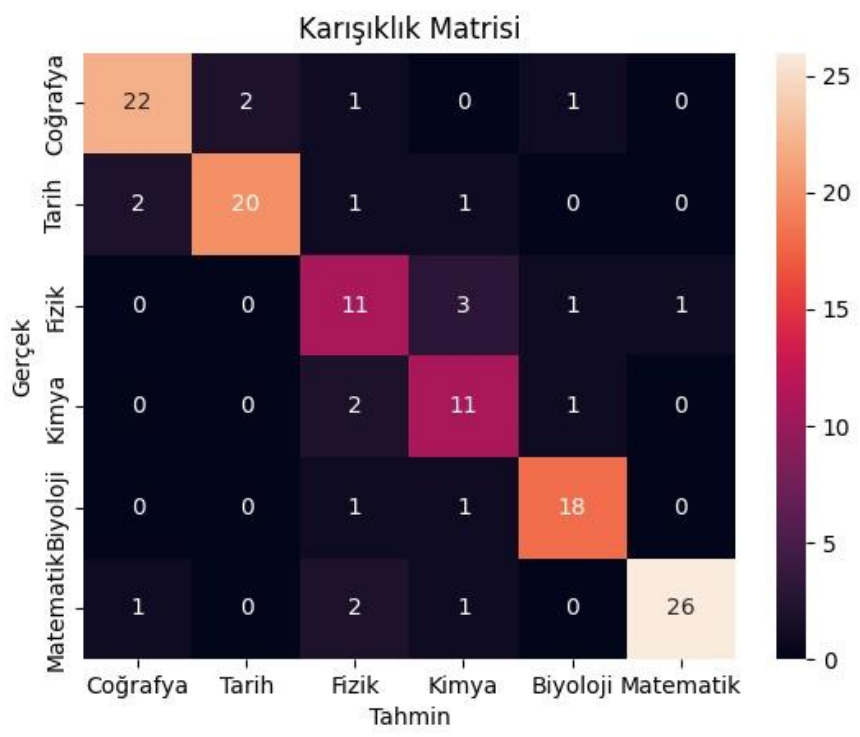

Şekil 8. Karlşılklk matrisi

Tablo 2. Nesne sayllarl ve doğruluk oranları

\begin{tabular}{lccccc}
\hline \multicolumn{1}{c}{ Konu } & Toplam & Doğruluk & Kesinlik & Duyarlılık & f1-score \\
\hline Coğrafya & 26 & 0,846 & 0.880 & 0.846 & 0,863 \\
\hline Tarih & 24 & 0.833 & 0.909 & 0.833 & 0.870 \\
\hline Fizik & 16 & 0.687 & 0.611 & 0.688 & 0.647 \\
\hline Kimya & 14 & 0.785 & 0.647 & 0.786 & 0.710 \\
\hline Biyoloji & 20 & 0.900 & 0.857 & 0.900 & 0.878 \\
\hline Matematik & 30 & 0.866 & 0.963 & 0.867 & 0.912 \\
\hline
\end{tabular}

\section{V.SONUC}

Artan içerik ve görselleştirme ihtiyaçları için otomatik sistemler kullanılmakta ve bunun için yapay zeka tekniklerinden faydalanılmaktadır. Derin öğrenme ve doğal dil işleme algoritmaları bunlardan bazılarıdır. Bu çalışmada da öğrenme nesnelerinin üstverilerinin KSA ve TSA ile görsel özelliklerinin metine dönüştürülmesi sağlanmaktadır. Oluşturulan metin üzerinde ise DDİ metotları uygulanarak IEEE LOM üstverisi doldurulmakta ve hangi konu alanına ait olduğu sinıflandırılmaktadır. Böylece üstveriler hızlı, kolay ve başarılı bir şekilde doldurulmakta ve bilgisayar gibi diğer sistemler tarafından otomatik olarak okunabilir ve kullanılabilir olmaktadır. Gelecek çalışmalarda otomatik olarak doldurulmuş ve sınıflandırılmış olan bu üstveriler kullanılarak otomatik içerik üretimi sağlanabilecektir.

\section{KAYNAKLAR}

[1] D. Jonassen, M. Davidson, M. Collins, J. Campbell, and B. B. Haag, "Constructivism and computer-mediated communication in distance education," American Journal of Distance Education, vol. 9, no. 2, pp. 7-26, 1995.

[2] Y. Beldarrain, "Distance education trends: integrating new technologies to foster student interaction and collaboration," Distance Education, vol. 27, no. 2, pp. 139-153, 2006.

[3] Ö. F. Bay ve H. Tüzün, "Yüksek öğretim kurumlarında ders içeriğinin web tabanlı olarak aktar1lmas1-I," Politeknik Dergisi, c. 5, s. 1, ss. 13-22, 2002. 
[4] T. Yigit, A. H. Isik, and M. Ince, "Web-based Learning object selection software using analytical hierarchy process," IET Software, vol. 8, no. 4, pp. 174-183, 2014.

[5] L. Becksford and S. Metko, "Using a library learning object repository to empower teaching excellence for distance students," Journal of Library \& Information Services in Distance Learning, vol. 12, pp. 120-129, 2018.

[6] K. Harman and A. Koohang, "Discussion board: a learning object," Interdisciplinary Journal of E-Learning and Learning Objects, vol. 1, pp. 67-77, 2005.

[7] J. Sinclair, M. Joy, Y. J. Yau, and S. Hagan, "A practice-oriented review of learning objects," IEEE Transactions on Learning Technologies, vol. 6, pp. 177-192, 2013.

[8] A. Zapata, V. H. Menéndez, M. E. Prieto, and C. Romero, "A framework for recommendation in learning object repositories: an example of application in civil engineering," Advances inEngineering Software, vol. 56, pp. 1-13, 2013.

[9] M. İnce, T. Yiğit, and A. H. Iş1k, "A hybrid AHP-GA method for metadata-based learning object evaluation," Neural Computing and Applications, vol. 31, no. 1, pp. 671-681, 2019.

[10] R. McGreal and T. Roberts, "A primer on metadata for learning objects: fostering an interoperable environment," E-learning, vol. 2, no. 10, pp. 26-29, 2001.

[11] P. Balatsoukas, A. Morris, and A. O'Brien, "Learning objects update: review and critical approach to content aggregation," Educational Technology \& Society, vol. 11, no. 2, pp. 119-130, 2008.

[12] G. Millar, Learning Objects 101: A Primer for Neophytes. Learning Resources Unit, British Columbia Institute of Technology, 2002.

[13] Y. Chen, "Educational resource management in grid community based on learning object metadata standard," International Journal of Emerging Technologies in Learning, vol. 13, no. 11, pp. 130-143, 2018.

[14] M. Knapp, Z. Risha, R. Gatewood, J. Van Der Volgen, R. Brown, and R. Kizilboga, "Learning to love the lor: implementing an internal learning object repository at a large national organization," Medical Reference Services Quarterly, vol. 38, no. 2, pp. 143-155, 2019.

[15] P. Brusilovsky, J. Eklund, and E. Schwarz, "Web-based education for all: a tool for development adaptive courseware," Computer Networks and ISDN Systems, vol. 30, no. 1, pp. 291 300, 1998.

[16] F. A. Dorça, R. D. Araújo, V. C. De Carvalho, D. T. Resend, and R. G. Cattelan, "An automatic and dynamic approach for personalized recommendation of learning objects considering students learning styles: an experimental analysis," Informatics in Education, vol. 15, no. 1, pp. 45-62, 2016.

[17] H. Imran, M. Belghis-Zadeh, T. W. Chang, and S. Graf, "PLORS: a personalized learning object recommender system," Vietnam Journal of Computer Science, vol. 3, no. 1, pp. 3-13, 2016.

[18] V. Dagiene, D. Gudoniene, and R. Bartkute, "The integrated environment for learning objects design and storing in semantic web," International Journal of Computers, Communications \&Control, vol. 13, no. 1, pp. 39-49, 2018. 
[19] F. Esposito, S. Ferilli, T. M. Basile, and N. Di Mauro, "Machine learning for digital document processing: from layout analysis to metadata extraction," in Machine learning in document analysis and recognition, Berlin, Heidelberg: Springer, 2008, pp. 105-138.

[20] S. Miranda and P. Ritrovato, "Supporting learning object repository by automatic extraction of metadata," Journal of e-Learning and Knowledge Society, vol. 11, no. 1, pp. 43-54, 2015.

[21] D. Roy, S. Sarkar, and S. Ghose, "Automatic extraction of pedagogic metadata from learning content," International Journal of Artificial Intelligence in Education, vol. 18, no. 2, pp. 97-118, 2008.

[22] E. Cortez, A. S. Da Silva, M. A. Gonçalves, F. Mesquita, and E. S. De Moura, "A flexible approach for extracting metadata from bibliographic citations," Journal of the American Society for Information Science and Technology, vol. 60, no. 6, pp. 1144-1158, 2009.

[23] K. Cardinaels, M. Meire, and E. Duval, "Automating metadata generation: the simple indexing interface," in Proceedings of the 14th International Conference on World Wide Web, 2005, pp. 548 556.

[24] H. Han, C. L. Giles, E. Manavoglu, H. Zha, Z. Zhang, and E. A. Fox "Automatic document metadata extraction using support vector machines," in Joint Conference on Digital Libraries Proceedings, 2003, pp. 37-48.

[25] P. Cimiano, S. Handschuh, and S. Staab, "Towards the self-annotating web," in Proceedings of the 13th International Conference on World Wide Web, 2004, pp. 462-471.

[26] B. Jebali and R. Farhat, "Ontology-based semantic metadata extraction approach," in International Conference on Electrical Engineering and Software Applications, 2013, pp. 1-5.

[27] W. Paik, S. Yilmazel, E. Brown, M. Poulin, S. Dubon, and C. Amice, "Applying natural language processing (nlp) based metadata extraction to automatically acquire user preferences," in Proceedings of the 1st International Conference on Knowledge Capture, 2001, pp. 116-122.

[28] P. Spinosa, G. Giardiello, M. Cherubini, S. Marchi, G. Venturi, and S. Montemagni, "NLP based metadata extraction for legal text consolidation," in Proceedings of the 12th International Conference on Artificial Intelligence and Law, 2009, pp. 40-49.

[29] R. Liu, L. Gao, D. An, Z. Jiang, and Z. Tang, "Automatic document metadata extraction based on deep networks," in National CCF Conference on Natural Language Processing and Chinese Computing, 2017, pp. 305-317.

[30] L. Deng and D. Yu, "Deep learning: methods and applications," Foundations and Trends ${ }^{\circledR}$ in Signal Processing, vol. 7, pp. 197-387, 2014.

[31] Y. LeCun, Y. Bengio, and G. Hinton, “Deep Learning,” Nature, vol. 521, no. 7553, pp. 436 $444,2015$.

[32] A. Krizhevsky, I. Sutskever, and G. E. Hinton, "Imagenet classification with deep convolutional neural networks," in Advances in Neural Information Processing Systems, 2012, pp.

1097-1105.

[33] J. Salamon and J. P. Bello, "Deep convolutional neural networks and data augmentation for environmental sound classification," IEEE Signal Processing Letters, vol. 24, no. 3, pp. 279-283, 2017. 
[34] Y. J. Cha, W. Choi, and O. Büyüköztürk, "Deep learning-based crack damage detection using convolutional neural networks," Computer-Aided Civil and Infrastructure Engineering, vol. 32, no. 5, pp. 361-378, 2017.

[35] O. Vinyals, A. Toshev, S. Bengio, and D. Erhan, "Show and tell: lessons learned from the 2015 mscoco image captioning challenge," IEEE Transactions on Pattern Analysis and Machine Intelligence, vol. 39, no. 4, pp. 652-663, 2016.

[36] A. V. D. Oord, N. Kalchbrenner, and K. Kavukcuoglu, "Pixel recurrent neural networks," 2016, arXiv:1601.06759.

[37] M. Schuster and K. K. Paliwal, "Bidirectional recurrent neural networks," IEEE Transactions on Signal Processing, vol. 45, no. 11, pp. 2673-2681, 1997.

[38] G. Toderici, D. Vincent, N. Johnston, S. Jin Hwang, D. Minnen, J. Shor, and M. Covell, "Full resolution image compression with recurrent neural networks," in Proceedings of the IEEE Conference on Computer Vision and Pattern Recognition, 2017, pp. 5306-5314.

[39] A. Graves, "Generating sequences with recurrent neural networks," 2013, arXiv:1308.0850.

[40] A. Graves and J. Schmidhuber, "Offline handwriting recognition with multidimensional recurrent neural networks," in Advances in Neural Information Processing Systems, 2009, pp. 545 552.

[41] T. Hughes and K. Mierle, "Recurrent neural networks for voice activity detection," in IEEE International Conference on Acoustics, Speech and Signal Processing, 2013, pp. 7378-7382.

[42] S. K. Metin, T. Kisla, and B. Karaoglan, "Named entity recognition in Turkish using association measures," Advanced Computing, vol. 3, no. 4, pp. 43-49, 2012.

[43] M. H. Stefanini and Y. Demazeau "TALISMAN: a multi-agent system for natural language processing," in Brazilian Symposium on Artificial Intelligence, 1995, pp. 312-322.

[44] S. Sun, C. Luo, and J. Chen, "A review of natural language processing techniques for opinion mining systems," Information Fusion, vol. 36, pp. 10-25, 2017.

[45] T. Strzalkowski, F. Lin, J. Wang, and J. Perez-Carballo, "Evaluating natural language processing techniques in information retrieval," in Natural Language Information Retrieval, Dordrecht: Springer, 1999, pp. 113-145.

[46] T. Nasukawa and J. Yi, "Sentiment analysis: capturing favorability using natural language processing," in Proceedings of the 2nd International Conference on Knowledge Capture, 2003, pp. $70-77$.

[47] Y. Aktaş, E. Y. İnce, and A. Çakır, "Doğal dil işleme kullanarak bilgisayar ağ terimlerinin wordnet ontolojisinde uyarlanmas1," Teknik Bilimler Dergisi, c. 7, s. 2, ss. 1-9, 2019.

[48] J. Cushing and R. Hastings, "Introducing computational linguistics with NLTK (natural language toolkit)," Journal of Computing Sciences in Colleges, vol. 25, no. 1, pp. 167-169, 2009.

[49] S. Savaş and N. Topaloğlu, "Data analysis through social media according to the classified crime," Turkish Journal of Electrical Engineering \& Computer Sciences, vol. 27, no. 1, pp. 407-420, 2019. 
[50] E. Y. İnce, "Spell checking and error correcting application for Turkish," International Journal of Information and Electronics Engineering, vol. 7, no. 2, pp. 68-71, 2017.

[51] M. N. Al-Kabi, T. M. Hailat, E. M. Al-Shawakfa, and I. M. Alsmadi, "Evaluating English to Arabic machine translation using BLEU," International Journal of Advanced Computer Science and Applications, vol. 4, no. 1, pp. 66-73, 2013.

[52] S. Stoll, N. C. Camgöz, S. Hadfield, and R. Bowden, "Text2Sign: towards sign language production using neural machine translation and generative adversarial networks," International Journal of Computer Vision, vol. 128, pp. 891-908, 2020.

[53] T. Sing, O. Sander, N. Beerenwinkel, and T. Lengauer, "ROCR: visualizing classifier performance in R," Bioinformatics, vol. 21, no. 20, pp. 3940-3941, 2005.

[54] M. Hossin and M. N. Sulaiman, "A review on evaluation metrics for data classification evaluations," International Journal of Data Mining \& Knowledge Management Process, vol. 5, no. 2, pp. 1-11, 2005.

[55] Coco. (2021, Aug 1). Ms-coco. [Online]. Available: https://cocodataset.org/\#home 